\title{
A rare case of lung abscess due to esophageo-pulmonary fistula in asymptomatic carcinoma of esophagus
}

\author{
Soumitra Ghosh ${ }^{1}$, Neha Handa ${ }^{2}$, Sudheer Tale ${ }^{1}$, Ashish Bhalla ${ }^{1}$ \\ ${ }^{1}$ Department of Internal Medicine, Post Graduate Institute of Medical Education and Research, Chandigarh; \\ ${ }^{2}$ Department of Nursing, Post Graduate Institute of Medical Education and Research, Chandigarh, India
}

\begin{abstract}
Acquired esophago-respiratory fistulae are usually esophagotracheal or esophago-bronchial. Esophago-pulmonary fistulae are rare. Most patients present with cardinal symptoms of esophageal carcinoma or esophago-pulmonary fistula leading to early diagnosis. We report a 56-years-old female with an unusual presentation. She presented with high grade fever with chills and rigor, cough with mucopurulent expectoration and shortness of breath for 15 days without dysphagia, nausea, vomiting or chest pain. Clinically and radiologically a diagnosis of lung abscess was entertained and
\end{abstract}

Correspondence: Soumitra Ghosh, Room Number 210, Block M, New Doctors Hostel, Post Graduate Institute of Medical Education and Research, Chandigarh, 160012, India.

Tel. +91 7908054090 .

E-mail: soumitra183@gmail.com

Authors' contributions: SG, NH, drafting the article, analysis and interpretation; ST, drafting the article and manuscript preparation; $\mathrm{AB}$, drafting the article and revising its intellectual content.

Acknowledgements: The authors would like to thank the patient for her willingness to be included in the study.

Conflict of interest: The authors declare no potential conflict of interest. Funding: None.

Informed consent: The authors declare that the patient's consent has been obtained for the publication of the paper.

Availability of data and materials: All data generated or analyzed during this study are included in this published article.

Key words: Lung abscess; esophageal cancer; esophageo-pulmonary fistula; self-expandable metallic stent.

Received for publication: 26 February 2021.

Accepted for publication: 28 April 2021.

${ }^{\circ}$ Copyright: the Author(s), 2021

Licensee PAGEPress, Italy

Monaldi Archives for Chest Disease 2021; 91:1815

doi: 10.4081/monaldi.2021.1815

This article is distributed under the terms of the Creative Commons Attribution Noncommercial License (by-nc 4.0) which permits any noncommercial use, distribution, and reproduction in any medium, provided the original author(s) and source are credited. she was treated with multiple antibiotics without any improvement. Contrast enhanced computed tomography (CECT) chest revealed esophageal malignancy with esophageal-pulmonary fistula communicating with abscess cavity. Patient responded to palliation with self-expandable esophageal stent and drainage of abscess. Although rare, asymptomatic malignant esophageal disease should be considered in the differential diagnosis of lung abscess, which does not follow a usual course.

\section{Introduction}

Acquired esophago-respiratory fistulae are a well-known complication of esophageal carcinoma, the majority of which are usually esophago-tracheal or esophago-bronchial fistula [1]. Esophageo-pulmonary fistulae have been rarely described [2-5]. In addition, all such reported patients either presented with dysphagia, vomiting or pain leading to diagnosis of esophageal carcinoma or cough with deglutition leading to diagnosis of esophageo-respiratory fistula [1]. In this article, we described a patient, who presented with necrotizing pneumonia of lower lobe of right lung without any esophageal symptoms. We did not think of such possibility initially. This type of presentation has been reported rarely in medical literature.

\section{Case Report}

A 56-year-old female presented at emergency department with high grade fever with chills and rigor, cough with mucopurulent expectoration and dyspnea for 15 days. She lost $10 \mathrm{~kg}$ weight in last two months. There was no history of dysphagia, vomiting or chest pain. She had rheumatoid arthritis and was taking homeopathic medicine (syrup containing rhus toxicodendron, bryonia, belladonna and other ingredients). She was investigated and treated in a clinic as pneumonia with oral amoxycilline-clavulinic acid for 7 days without any improvement. On presentation at our hospital, she was febrile with temperature of $102.5^{\circ} \mathrm{F}$, had tachycardia with heart rate of $120 / \mathrm{min}$ and tachypnea with respiratory rate of $24 / \mathrm{min}$. Her blood pressure was $136 / 80 \mathrm{~mm}$ of $\mathrm{Hg}$ and $\mathrm{SpO}_{2}$ of $92 \%$ at room air. On respiratory system examination, bronchial breath sound was heard on right middle and lower zone with coarse crepitation on auscultation. Other systemic examination was normal.

Blood investigations showed hemoglobin of $11.6 \mathrm{~g} / \mathrm{dL}$, total leucocyte count (TLC) of 24300 with $86 \%$ neutrophil with normal platelet count. Routine urine examination, renal function test and 
liver function test were normal except low albumin level $(2.3 \mathrm{~g} / \mathrm{dL})$. Initial chest X-ray revealed inhomogeneous opacity with cavitation and air-fluid level in the right lower zone indicating lung abscess (Figure 1). Gram stain of sputum examination showed mixed flora. She was started on piperacillin-tazobactum and clindamycin.

CECT chest done on next day revealed circumferential irregular mural thickening of middle $1 / 3^{\text {rd }}$ of esophagus (maximum 8 $\mathrm{mm}$ ), involving $4.5 \mathrm{~cm}$ long segment along with a thick-walled, well-defined collection of $7.7 \times 6.7 \times 6.7 \mathrm{~cm}$ with internal air-fluid level and a tongue like extension of this collection extended into posterior mediastinum, closely abutting and displacing the esophagus (Figure 2A). On oral contrast administration, a $3 \mathrm{~mm}$ sized defect was seen in the right lateral wall of esophagus, leaking the contrast into the collection indicating esophageo-pulmonary fistula (Figure 2B). A $2.4 \times 2.4 \mathrm{~cm}$ sized lesion with speculated margins seen in right breast which was likely metastatic deposit. Multiple pre-tracheal, pre-vascular and bilateral hilar lymphadenopathy were also noted.

Fluoroscopy-guided percutaneous drainage of the abscess content by intervention radiologist was done on the same day. Under local anesthesia, a large bore 12 French drainage catheter was placed at the most dependent portion inside the abscess cavity. Content inside the cavity was drained and it was irrigated with normal saline.

By fourth day of hospitalization, there was some improvement clinically and radiologically on chest X-ray. She became afebrile. Cultures of sputum and abscess content were subsequently found to be sterile.

Upper gastro-intestinal endoscopy (UGIE) was done which revealed segmented ulcerated lesions in the mid esophagus. Multiple biopsies were taken and a nasogastric tube (NG tube) was placed over the guidewire. General surgery consultation was done but considering advanced disease with possible metastasis, they denied any operative treatment. On next day, under fluoroscopic guidance, $18 \mathrm{~mm}$ x $150 \mathrm{~mm}$ covered self-expandable metallic stent (SEMS) (Ultraflex Esophageal NG, Boston Scientific, Marlborough, USA) was placed to cover the lesion for palliative treatment (Figure 3).

Later, biopsy was reported as moderately differentiated squamous cell carcinoma. Patient was categorized as advanced disease with stage IVB (T4b N2 M1). Treatment option was only palliation

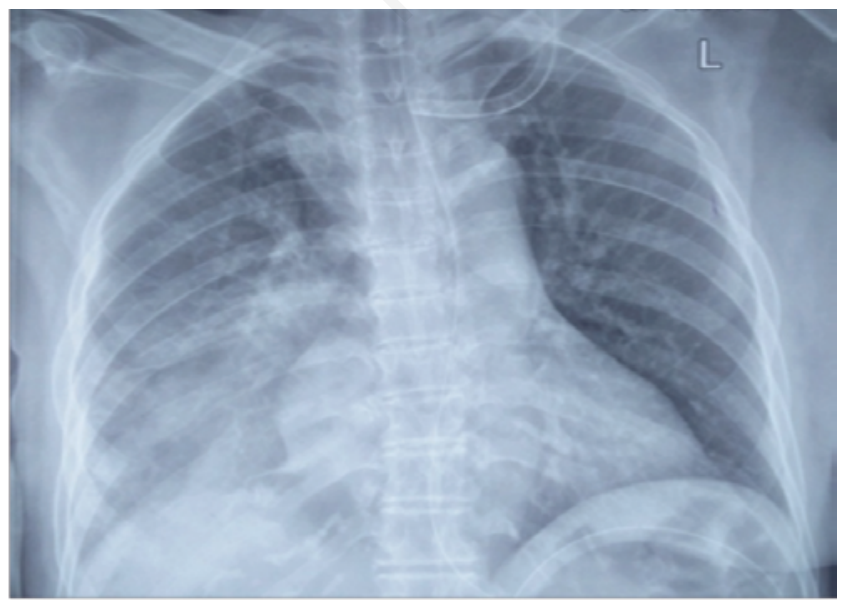

Figure 1. Chest X-ray showing inhomogeneous opacity with cavitation and air-fluid level in the right lower zone.
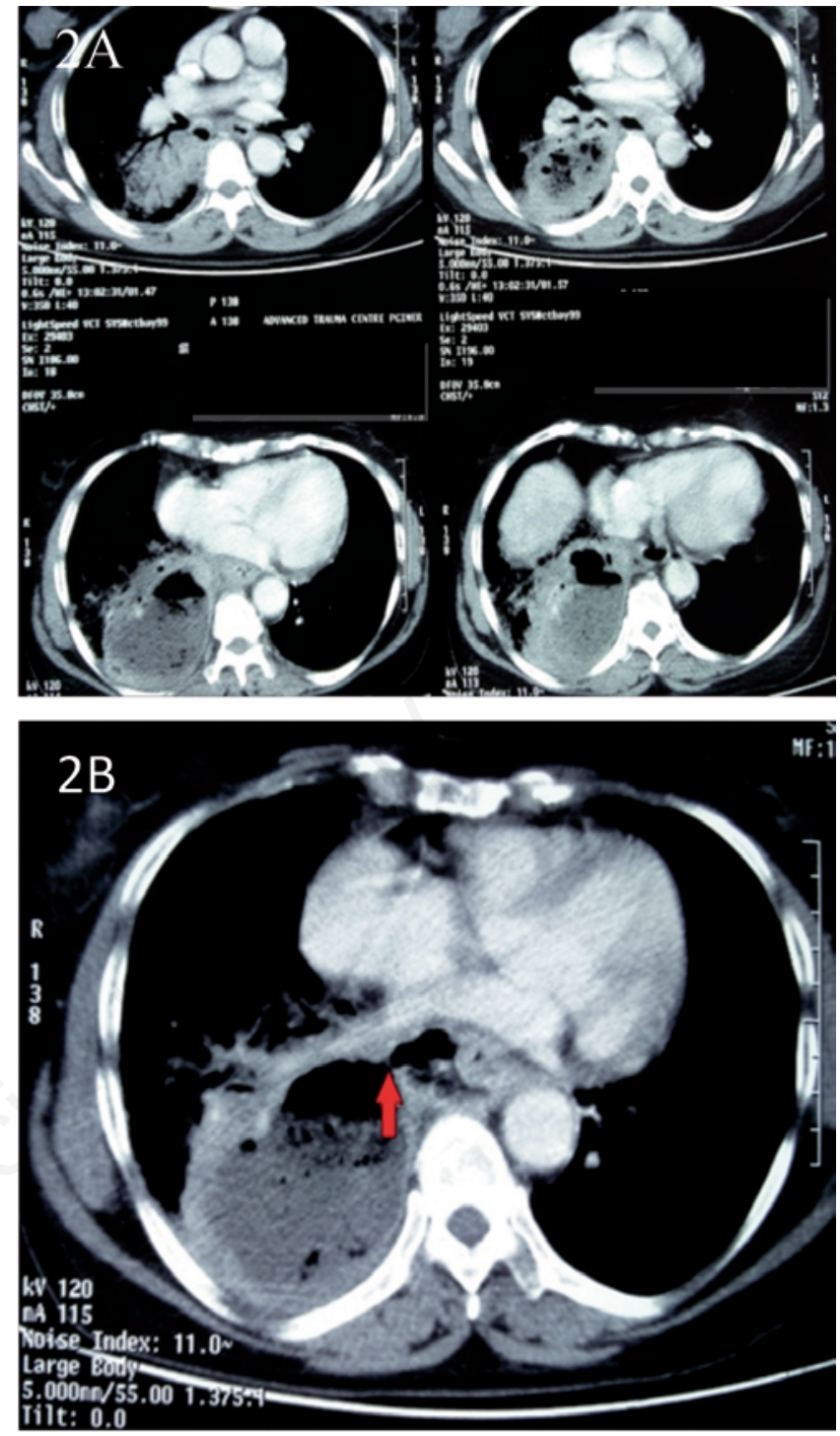

Figure 2. a) Well-defined collection of $7.7 \times 6.7 \times 6.7 \mathrm{~cm}$ with internal air-fluid level in the posterior mediastinum suggestive of lung abscess. b) On oral contrast administration, a $3 \mathrm{~mm}$ sized defect (red arrow) seen in the right lateral wall of esophagus, leaking the contrast into the collection which indicate esophageo-pulmonary fistula.

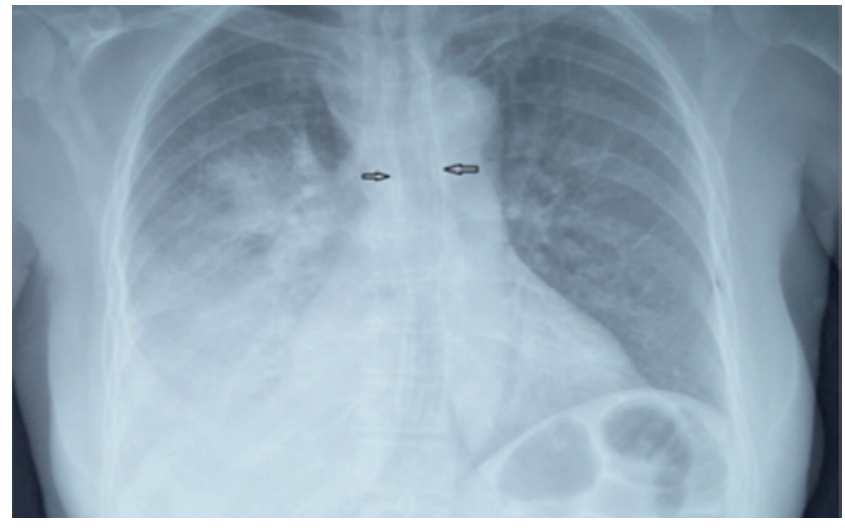

Figure 3. Covered esophageal self-expandable metallic stent (SEMS) was placed (arrow). 
with chemotherapy or chemoradiation therapy. She was referred to radiotherapy department for cisplatin-based chemotherapy and radiotherapy.

She was discharged with oral antibiotics on ninth day. At the time of discharge, she was afebrile, cough subsided, shortness of breath improved and TLC decreased to 14000 . On four months follow-up, she was doing better with no residual cavity in lung parenchyma.

\section{Discussion}

Esophago-respiratory fistulae are uncommon and may be congenital or acquired. Acquired esophago-respiratory fistulae are usually secondary to malignancy, prolonged intubation, corrosive ingestion, granulomatous mediastinal infections, post-surgery on the esophagus or trachea-bronchial tree, trauma or radiotherapy [1]. Esophago-respiratory fistulas are seen in 5-13\% cases of esophageal cancer. Most esophago-respiratory fistulae are due to an esophago-tracheal fistula or an esophago-bronchial fistula [6]. Esophago-pulmonary fistulae without tracheal or bronchial involvement have been rarely reported [2-5].

Esophageal cancers may exhibit highly aggressive local growth which may lead to multiple pulmonary fistulae causing pneumonitis and lung abscesses. This may lead to sepsis, acute respiratory distress syndrome (ARDS) and death [3-4].

Our patient had no cardinal symptoms of esophageal carcinoma like dysphagia, pain, regurgitation, vomiting or symptoms of esophageal-respiratory fistula like coughing with swallowing food. Retrospectively anorexia and weight loss could be a clue for malignancy, but it could be attributed to chronic infection like lung abscess as well. This diagnosis was not initially suspected in our patient. Francis and Goldstein reported one similar case of asymptomatic esophageal carcinoma with esophageo-pulmonary fistula masquerading as a primary lung abscess [6].

To prove esophageo-respiratory fistula, it is necessary to demonstrate the fistulous tract, as contrast material within the bronchial lumen or parenchymal cavity may be due to aspiration. Multidetector computed tomography (MDCT) and 3-D reconstruction help in the diagnosis and assessment of fistula, and is useful in planning interventions [4]. In our patient, oral contrast administration during CECT chest showed contrast leaking into the lung cavity through a defect in the right lateral wall of esophagus, thus proving it to be esophageal-pulmonary fistula. Another novel way of diagnosis of fistula tract is by video-fluoroscopic swallowing study (VFSS). VFSS captures serial video-radiographic images of barium-coated foods or liquids when they pass through the mouth, pharynx and esophagus in real time [7].

Malignant esophago-respiratory fistulae have no curative treatment. Palliative procedures like esophageal stenting, bypass, exclusion or surgical repair with fistula resection are the only option and they may prolong survival [8]. Radiotherapy or chemotherapy may also give some palliation. Mean survival is only one to six weeks without treatment [9]. Endoscopic esophageal stenting with covered self-expandable metal stents (SEMS) is currently most widely used palliative treatment modal- ity. Along with placement of SEMS, it is very important to treat lung abscess simultaneously with antibiotics and drainage because such closure hinders natural drainage of lung abscesses into the esophagus, thus exacerbating the severity of the lung abscess [5]. According to some authors, surgical treatment with resection of the esophageal lesion and the involved pulmonary lobe might significantly prolong the survival in these patients [10-12].

\section{Conclusions}

Our case is unusual, in the sense that despite the presence of esophago-pulmonary fistula, communicating with lung abscess cavity, there were no symptoms commonly associated with esophageal carcinoma or esophago-pulmonary fistula. Although rare, asymptomatic malignant esophageal disease should be considered in the differential diagnosis of lung abscess, which does not follow a usual course.

\section{References}

1. Duranceau A, Jamieson GG. Malignant tracheo-esophageal fistula. Ann Thorac Surg 1984;37:346-4.

2. Leguen Y, Stern JB, Sauvanet A, et al. Esophageal pulmonary fistula in squamous cell carcinoma of the esophagus. Rev Mal Respir 2000;17:965-8.

3. Satija L, Joshi P, George R, et al. An unusual case of malignant esophago-pulmonary fistula diagnosed by multidetector computed tomography. Med J Armed Forces India 2012;68:72-4.

4. Kim KR, Shin JH, Song HY, et al. Palliative treatment of malignant esophago-pulmonary fistulas with covered expandable metallic stents. AJR Am J Roentgenol 2009;193:278-2.

5. Francis PB, Goldstein J. Asymptomatic esophageal carcinoma with esophago-pulmonary fistula masquerading as a primary lung abscess. South Med J 1979;72:75-7.

6. Fitzgerald RH, Jr, Bartels DM, Parker EF. Tracheo-esophageal fistulas secondary to carcinoma of the esophagus. J Thorac Cardiovasc Surg 1981;82:194-7.

7. Martin-Harris B, Jones B. The video-fluorographic swallowing study. Phys Med Rehabil Clin N Am 2008;19:769-85.

8. Shin JH, Song HY, Ko GY, et al. Esophago-respiratory fistula: long-term results of palliative treatment with covered expandable metallic stents in 61 patients. Radiology 2004; 232:252-9.

9. Sebastian J, Kirankumar VS, Pappachan JM, et al. Multifactorial dysphagia complicated by esophago-bronchial fistula. J Cancer Res Ther 2007;3:108.

10. Kato H, Tachimori Y,Watanabe H, Itabashi M. Surgical treatment of thoracic esophageal carcinoma directly invading the lung. Cancer 1992;70:1457-1.

11. Davydov M, Stilidi I, Bokhyan V, Arzykulov G. Surgical treatment of esophageal carcinoma complicated by fistulas. Eur J Cardiothorac Surg 2001;20:405-8.

12. Rehders A, Baseras B, Telan L, et al. Malignant esophago-pulmonary fistula. Thor Cancer 2014;5 468-471. 